\title{
Validation study of postoperative liver failure and mortality risk scores after liver resection for perihilar cholangiocarcinoma
}

\author{
Takehiro Noji1" ${ }^{1 \#}$, Satoko Uemura ${ }^{1 \#}$, Jimme K. Wiggers ${ }^{2}$, Kimitaka Tanaka ${ }^{1}$, Yoshitsugu Nakanishi ${ }^{1}$, \\ Toshimichi Asano ${ }^{1}$, Toru Nakamura ${ }^{1}$, Takahiro Tsuchikawa ${ }^{1}$, Keisuke Okamura ${ }^{1}$, Pim B. Olthof ${ }^{2,3}$, \\ William R. Jarnagin ${ }^{4}$, Thomas M. van Gulik², Satoshi Hirano ${ }^{1}$
}

${ }^{1}$ Department of Gastroenterological Surgery II, Hokkaido University Faculty of Medicine, Sapporo, Japan; ${ }^{2}$ Department of Surgery, Cancer Center Amsterdam, Amsterdam UMC, Location AMC University of Amsterdam, Amsterdam, the Netherlands; ${ }^{3}$ Department of Surgery, Erasmus Medical Center, Rotterdam, the Netherlands; ${ }^{4}$ Department of Surgery, Memorial Sloan Kettering Cancer Center, New York, NY, USA

Contributions: (I) Conception and design: T Noji, TM van Gulik, JK Wiggers, PB Olthof; (II) Administrative support: None; (III) Provision of study materials or patients: None; (IV) Collection and assembly of data: T Noji, S Uemura, K Tanaka, K Okamura; (V) Data analysis and interpretation: T Noji, S Uemura, JK Wiggers; (VI) Manuscript writing: All authors; (VII) Final approval of manuscript: All authors.

\#These authors contributed equally to this work.

Correspondence to: Takehiro Noji, MD, PhD. Department of Gastroenterological Surgery II, Hokkaido University Faculty of Medicine, North-15, West-7, Kita-ku, Sapporo 060-8638, Japan. Email: drnoji@med.hokudai.ac.jp.

Background: Surgery for perihilar cholangiocarcinoma (PHCC) remains a challenging procedure with high morbidity and mortality. The Academic Medical Center (Amsterdam UMC) and Memorial Sloan Kettering Cancer Center proposed a postoperative mortality risk score (POMRS) and post-hepatectomy liver failure score (PHLFS) to predict patient outcomes. This study aimed to validate the POMRS and PHLFS for PHCC patients at Hokkaido University.

Methods: Medical records of 260 consecutive PHCC patients who had undergone major hepatectomy with extrahepatic bile duct resection without pancreaticoduodenectomy at Hokkaido University between March 2001 and November 2018 were evaluated to validate the PHLFS and POMRS.

Results: The observed risks for PHLF were $13.7 \%, 24.5 \%$, and $39.8 \%$ for the low-risk, intermediaterisk, and high-risk groups, respectively, in the study cohort. A receiver-operator characteristic (ROC) analysis revealed that the PHLFS had moderate predictive value, with an analysis under the curve (AUC) value of 0.62. Mortality rates based on the POMRS were $1.7 \%, 5 \%$, and $5.1 \%$ for the low-risk, intermediate-risk, and high-risk groups, respectively. The ROC analysis demonstrated an AUC value of 0.58 .

Conclusions: This external validation study showed that for PHLFS the threshold for discrimination in an Eastern cohort was reached (AUC >0.6), but it would require optimization of the model before use in clinical practice is acceptable. The POMRS were not applicable in the eastern cohort. Further external validation is recommended.

Keywords: Perihilar cholangiocarcinoma (PHCC); post operative mortality score; post-hepatectomy liver failure score (PHLFS); validation study

Submitted Aug 12, 2020. Accepted for publication Nov 13, 2020.

doi: $10.21037 / \mathrm{hbsn}-20-660$

View this article at: http://dx.doi.org/10.21037/hbsn-20-660

\section{Introduction}

Surgery for perihilar cholangiocarcinoma (PHCC) is challenging because of its high morbidity and mortality despite elaborate multidisciplinary perioperative management $(1,2)$. Morbidity and mortality rates after surgery for patients with PHCC have been reported to range from $26 \%$ to $68 \%$ and $1.4 \%$ to $18 \%$, respectively, 
Table 1 Five risk factors and assigned points in PHCC patients based on the AMC/MSKCC scoring system

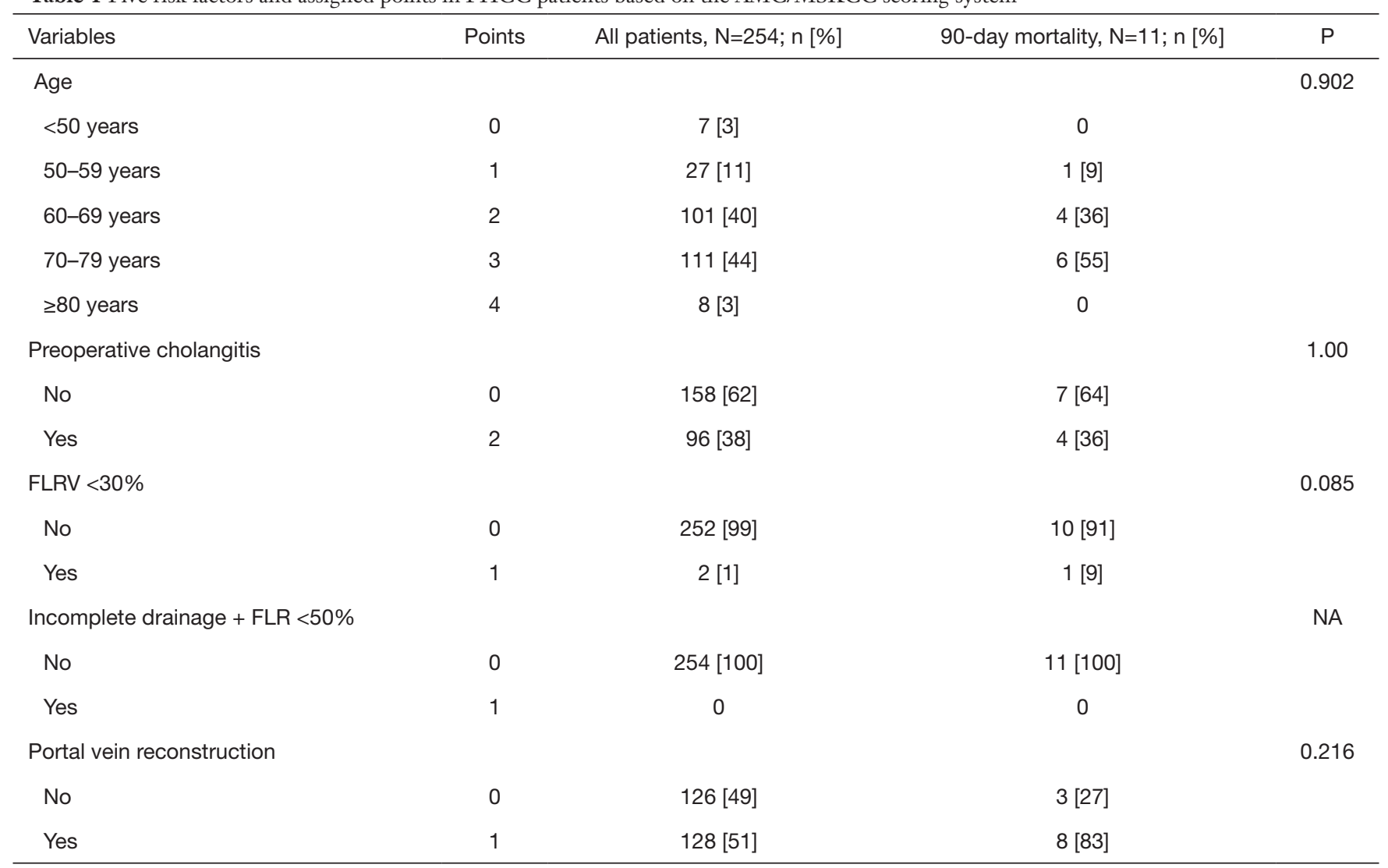

PHCC, perihilar cholangiocarcinoma; AMC/MSKCC, Academic Medical Center/Memorial Sloan Kettering Cancer Center; FLR, future liver remnant; NA, not assessed.

thus exceeding those of other hepato-biliary procedures (2). Previous reports have shown that post-hepatectomy liver failure (PHLF) is a common and serious complication, with a reported incidence of $22 \%$ to $33 \%$ and high mortality rates of $52 \%$ to $68 \%$ (3-6). Patients with PHCC usually have obstructive cholestasis and ultimately, jaundice due to the location of the tumour at the liver hilum (7-9). Cholestasis impairs the regenerative capacity of the liver after resection, which increases surgical risk, especially when the future liver remnant (FLR) volume (FLRV) is small $(6,10,11)$. Therefore, it is essential to select candidates for PHCC surgery based not only on the tumour status but also on the patients' preoperative condition.

In 2016, two Western high-volume centres for PHCC [the Academic Medical Center of Amsterdam UMC (AMC) and the Memorial Sloan Kettering Cancer Center (MSKCC) in New York] proposed a postoperative mortality risk score (POMRS) for PHCC (12). They evaluated 228 PHCC patients and determined that postoperative mortality at 90 days was independently predicted by age, preoperative cholangitis, FLRV $<30 \%$, portal vein reconstruction (PVR), and incomplete FLRV drainage in patients with a FLRV volume $<50 \%$ (Table 1). In 2017, the same team also proposed a PHLF risk score (PHLFS) (3). They evaluated a consecutive series of 217 patients who underwent major liver resection for PHCC. They showed that the risk factors for PHLF were FLRV volume $<30 \%$ (small), FLRV of $30 \%$ to $45 \%$ (intermediate volume), jaundice at presentation, immediate preoperative bilirubin $>50 \mathrm{mmol} / \mathrm{L}(>2.9 \mathrm{mg} / \mathrm{dL})$, and preoperative cholangitis (Table 2). However, no external validation studies for both the POMRS and PHLFS have been reported.

Previous studies have reported significant differences in treatment and outcomes of Western (e.g., Europe, Northern America) and (South-East) Asian centres (e.g., Japan, Korea, and China) (2). To determine the differences 
Table 2 Risks predicted by the PHLF risk score of Hokkaido University and AMC/MSKCC

\begin{tabular}{|c|c|c|c|c|c|c|c|}
\hline Group & Total points & \multicolumn{3}{|c|}{ Hokkaido University } & \multicolumn{3}{|c|}{ AMC/MSKCC } \\
\hline \multirow[t]{2}{*}{ Low risk } & 0 & $40 / 6$ & 4 & 13.7 & 34 & 4 & 5 \\
\hline & 1 & $11 / 1$ & & & 10 & 7 & \\
\hline \multirow[t]{3}{*}{ High risk } & 4 & $65 / 22$ & 44 & 39.8 & 53 & 33 & 44 \\
\hline & 5 & $27 / 14$ & & & 18 & 48 & \\
\hline & 6 & $1 / 1$ & & & 11 & 64 & \\
\hline
\end{tabular}

PHLF, post-hepatectomy liver failure; AMC/MSKCC, Academic Medical Centre/Memorial Sloan Kettering Cancer Center.

between Western and Asian PHCC treatments, we recently performed a comparative study (13). This study showed, despite more aggressive surgery (with concomitant vascular resection) undertaken in the Asian cohort, that the 90-day mortality rate was lower (Asian cohort, 7\%; Western cohort, $14 \% ; \mathrm{P}=0.066)$. This discrepancy could be explained by differences in patients' characteristics, such as the American Society of Anaesthesiologists (ASA) score, use of preoperative biliary drainage, and indication for portal vein embolization (PVE).

The results also raised the clinical question whether the PHLFS and POMRS, which were established based on Western-centre cohorts with patient backgrounds different from those in Asia, can be applied to a cohort of a South-East Asian-centre. The objective of this study was to validate the POMRS and PHLFS for PHCC patients at Hokkaido University, which is a high-volume centre for PHCC in Japan. We present the following article in accordance with the STROBE reporting checklist (available at https://hbsn.amegroups.com/article/view/10.21037/ hbsn-20-660/rc).

\section{Methods}

\section{Ethics}

The study was approved by the institutional review board of Hokkaido University (No. 018-0429) and conducted according to the Declaration of Helsinki (as revised in 2013). It was registered at the UMIN-CTR (No. UMIN000036229).

\section{Data collection}

This retrospective study was conducted from March 2019 to June 2019.

\section{Patients}

The PHLFS and POMRS were validated for 260 consecutive PHCC patients subjected to major hepatectomy with extrahepatic bile duct resection without pancreaticoduodenectomy at Hokkaido University, Dept. of Gastroenterological Surgery II between March 2001 and November 2018.

\section{Control group}

To compare with previous results of the PHLFS and POMRS, we referred to published data of AMC/MSKCC's PHLFS and POMRS $(3,12)$.

\section{Preoperative diagnosis}

The details of preoperative diagnosis of PHCC have been described previously $(6,7,14,15)$. The diagnosis of PHCC was based on clinical presentation, imaging studies using 
enhanced computed tomography (CT), magnetic resonance imaging (MRI), direct cholangiography, and biopsy.

\section{Biliary drainage}

Biliary drainage procedures have been described in previous reports (7). The aim of preoperative biliary drainage at Hokkaido University was complete drainage of the FLRV. All PHCC patients had undergone surgery with complete drainage of the FLRV at Hokkaido University (6). Preoperative biliary decompression was performed to reduce the serum bilirubin concentration to $<2 \mathrm{mg} / \mathrm{dL}$ in all patients with jaundice and to control segmental cholangitis. Before 2005, single or multiple percutaneous transhepatic biliary drainage (PTBD) was performed (14). As of 2005, selective single or double endoscopic naso-biliary drainage (ENBD) of the FLR was adopted as the initial drainage procedure. Additional second ENBD catheters were placed to drain the biliary tree in future remnant livers that were not decompressed by the first catheter or to control cholangitis when this occurred. Alternatively, PTBD was used when drainage with ENBD was not effective or if a third catheter was required (16).

\section{Cholangitis}

Preoperative cholangitis was defined when all of the following criteria were fulfilled: fever with body temperature higher than $38^{\circ} \mathrm{C}$, decrease of temperature after new placement of a drainage catheter, and/or liver abscess formation with fever.

\section{Assessment of the remnant liver function, portal vein embolization, and surgical indications}

Liver function of all patients was evaluated preoperatively using methods similar to those used by the Nagoya University group (17). To evaluate whole liver function, an indocyanine green (ICG) regression test was performed before surgery (6). The plasma disappearance rate of ICG (ICGK) was calculated using a linear regression analysis of plasma ICG concentration, as previously described $(6,17)$. FLR plasma clearance rate of ICG (ICGK-F) values were calculated by multiplying ICGK by the proportion of the FLRV. The proportion of the FLRV was calculated by CTvolumetry, which was also performed preoperatively.

Preoperative PVE of the liver lobe to be resected was indicated when subsequent right hepatectomy, right trisectionectomy, or left trisectionectomy was planned $(15,18)$. Methods for PVE at our institution have been described previously (19). Two or 3 weeks after PVE, the ICGK-F was re-evaluated and surgery was performed. When the ICGK-F value did not fulfil the Nagoya criteria $(\geq 0.05)$ (17), surgery was postponed.

\section{Surgery}

The surgical procedures for PHCC have been described previously $(14,18,20,21)$. Briefly, radical resection included en bloc excision of the liver hilum with (extended) hemihepatectomy, including the caudate lobe in most cases, complete lymphadenectomy of the hepatoduodenal ligament, and excision and reconstruction of each or both the portal vein and hepatic artery if necessary. The portal vein was reconstructed by HPB surgeons; however, the hepatic artery was reconstructed by plastic surgeons (until 2015) or cardiovascular surgeons (from 2016 to present).

\section{Definition of complications}

Postoperative complications consistent with Clavien-Dindo classification $\geq$ IIIa were defined as morbidity (22). Ninetyday in-hospital death after surgery was defined as mortality. PHLF was defined as International Study Group of Liver Surgery (ISGLS) grade B or C (23).

\section{Statistical analysis}

Statistical calculations were performed using JMP version 14 (SAS, Cary, NC, USA), and statistical analyses were performed using SPSS version 22 (IBM Corp., Armonk, NY, USA) and R version 2.15 .3 (http://www.r-project.org/) software packages.

The chi-square test, Fisher's exact test, and MannWhitney $\mathrm{U}$ test were used as appropriate. $\mathrm{P}<0.05$ was considered significant. To develop POMRS and PHLFS at Hokkaido University, cases were calculated based on the statistical methods previously used by AMC/MSKCC. The AUC value was calculated to estimate how well the model discriminated between patients with and without postoperative mortality or PHLF.

\section{Results}

Six patients were excluded from the study because of missing preoperative liver volume data; thus, 254 
Table 3 Patient characteristics in univariate analysis of post hepatectomy liver failure (PHLF) in the Hokkaido University series

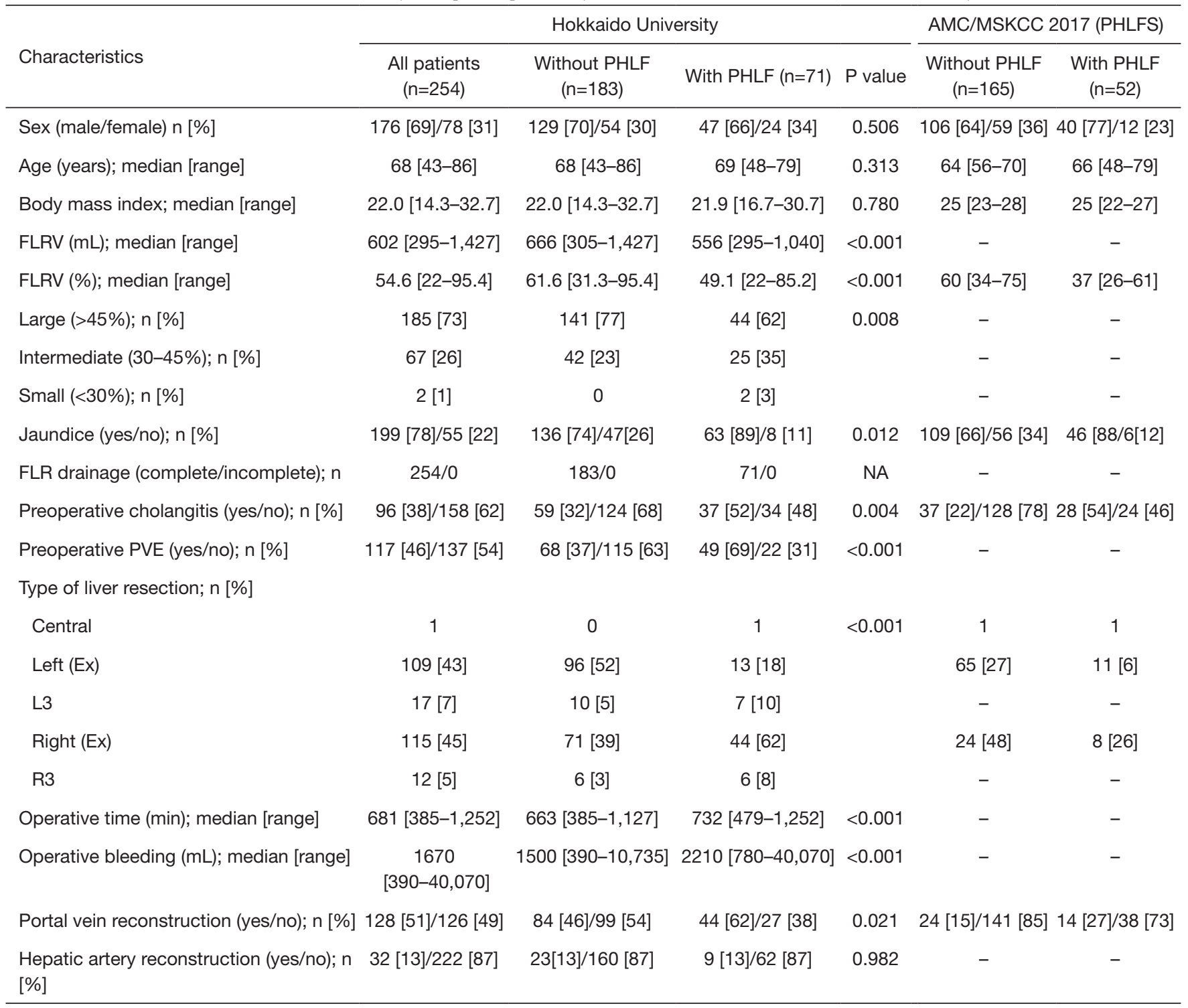

Values are presented as $\mathrm{n}$ or median (range in Hokkaido University; and interquartile range in PHLFS). "-"; not shown; PHLF, post hepatectomy liver failure; FLRV, future liver remnant volume; FLR, future liver remnant; PVE, portal vein embolization; central, central hepatectomy; left, left hepatectomy; L3, left trisectionectomy; right, right hepatectomy; R3, right trisectionectomy; Ex, extended hepatectomy including trisectionectomy; AMC, Academic Medial Center; MSKCC, Memorial Sloan Kettering Cancer Center; PHLFS, post hepatectomy liver failure score (Olthof et al. 2017).

patients with PHCC were included in the analysis. The postoperative pathological examination confirmed PHCC in all 254 patients. Table 3 shows the characteristics of the patients involved in this study and the characteristics of patients with or without PHLF.

The preoperative status, such as FLRV (percentage or volume), jaundice at presentation, and preoperative cholangitis, as well as intraoperative factors, such as
PVR, longer operative time, and/or higher volume of operative bleeding, were associated with PHLF (Table 3). The incidence of PHLF was almost similar in the AMC/ MSKCC and Hokkaido University cohorts (i.e., 24\% and $28 \%$, respectively; $\mathrm{P}=0.345)$. None of the patients in the Hokkaido University cohort had preoperative bilirubin levels exceeding $50 \mu \mathrm{mol} / \mathrm{L}(2.9 \mathrm{mg} / \mathrm{dL})$.

The risks predicted by the PHLFS for the AMC/ 
MSKCC and Hokkaido University cohorts are shown in Table 2. The predicted risk score for the Hokkaido University cohort was almost the same as that of the AMC/MSKCC cohort; the observed risks for PHLF in the Hokkaido University cohort were $13.7 \%, 24.5 \%$, and $39.8 \%$ for the low-risk, intermediate-risk, and high-risk groups, respectively. Receiver-operator characteristic (ROC) analysis revealed that the PHLFS had moderate predictive value, as demonstrated by an analysis of the AUC value of 0.62 for PHLF in the Hokkaido University cohort.

Next, we evaluated POMRS. The univariate analysis revealed that FLRV, operative time, operative bleeding, and PHLF influenced 90-day mortality in the Hokkaido University series (Table 4). However, patient age, preoperative cholangitis, and PVR, which were independent risk factors for 90-day mortality in the POMRS, were not associated with 90-day mortality in the Hokkaido University cohort (Table 1). Mortality rates based on POMRS in this cohort were $1.7 \%, 5 \%$, and $5.1 \%$ for the low-risk $(\mathrm{n}=59)$, intermediate-risk $(\mathrm{n}=120)$, and high-risk $(\mathrm{n}=75)$ patients, respectively (Table 5). The ROC analysis revealed that the POMRS was not useful for predicting the 90-day mortality in the Hokkaido University cohort, as demonstrated by an AUC value of 0.58 .

\section{Discussion}

In this study, the PHLFS and POMRS, which were established in Western high-volume centres for PHCC, were validated in an Asian cohort. The results showed that the PHLFS was only partially represented in the South-East Asian, high volume centre cohort whereas the POMRS was not applicable in this cohort.

PHLF is the most severe, life-threatening complication after PHCC surgery $(2,3,8)$. Although there were differences in patient and treatment characteristics in the Asian and Western cohorts, as determined previously (12), the present study showed that the incidences of PHLF were similar in both groups. A previous systematic review also supports this result (2).

The finding that this validation study did not completely reproduce previous results is possibly explained by several factors. First, amongst the four risk factors of PHLFS, jaundice at presentation and preoperative cholangitis were also risk factors for PHLF in the Hokkaido University cohort. However, the other two risk factors, FLRV $<30 \%$ and immediate preoperative bilirubin $>50 \mu \mathrm{mol} / \mathrm{L}$ $(2.9 \mathrm{mg} / \mathrm{dL})$, were not identified as risk factors. Very few patients had FLRV $<30 \%(2 / 254)$ and none had an immediate preoperative bilirubin level $>50 \mu \mathrm{mol} / \mathrm{L}$ $(2.9 \mathrm{mg} / \mathrm{dL})$ at Hokkaido University. As mentioned above, preoperative biliary drainage was performed routinely in jaundiced patients, and surgery for PHCC was not performed for patients with an preoperative bilirubin level $>34.2 \mu \mathrm{mol} / \mathrm{L}(2 \mathrm{mg} / \mathrm{dL})$ at Hokkaido University. Second, PVR was one of the risk factors in the Hokkaido University cohort, but it was not a risk factor in the PHLFS. PVR is associated with longer operative times and higher volumes of operative blood loss, which could be associated with PHLF. Third, PVE was associated with PHLF in the Hokkaido University cohort (Table 3). In general, PVE is also associated with a small future remnant liver and the type of hepatectomy, and such patients are also at higher risk for PHLF $(6,15)$. Therefore, high-risk patients are under-represented in many high volume Asian centres including Hokkaido University (1), and these differences between Asia and the West potentially lead to a relatively lower AUC level (0.62).

One important problem is the lack of a definitive definition of PHLF for PHCC surgery $(6,24)$. Recently, Kawamura et al. reported that the ISGLS criteria are inferior to other grading systems for PHLF after PHCC surgery, such as the maximum total bilirubin level criteria (maximum total bilirubin level $>7 \mathrm{mg} / \mathrm{dL}$ on postoperative day 7), 3-4-50 criteria (total bilirubin $>4 \mathrm{mg}$ and prothrombin time $<50 \%$ on postoperative day 3 ), and 50-50 criteria for predicting PHLF-related mortality (6). This study showed that when PHLF was defined using the ISGLS criteria for PHCC surgery, it showed high sensitivity but had low specificity for PHLF-related death. This was also shown in our results presented in Table 2. It appears that the PHLFS underpredicted PHLF in the low and intermediate risk groups in the Hokkaido cohort, but was predictive in the high risk. Although the reasons for this discrepancy are uncertain, we suspect that the use of the ISGLS criteria led to false positive cases in patients with low or intermediate risk. Furthermore, because there are significant differences between regular hepatectomies and large resections for PHCC-the latter requiring major hepatectomy with extra-hepatic bile duct resection and lymphadenectomy $(25,26)$ - further validation studies using a modified PHLF definition are needed to confirm the usefulness of the PHLFS.

The POMRS consisted of five patient characteristics: age, preoperative cholangitis, FLRV $(<30 \%)$, incomplete drainage + FLRV $<50 \%$, and PVR (12); however, these risk 
Table 4 Univariate analysis of 90-day mortality in the Hokkaido University series

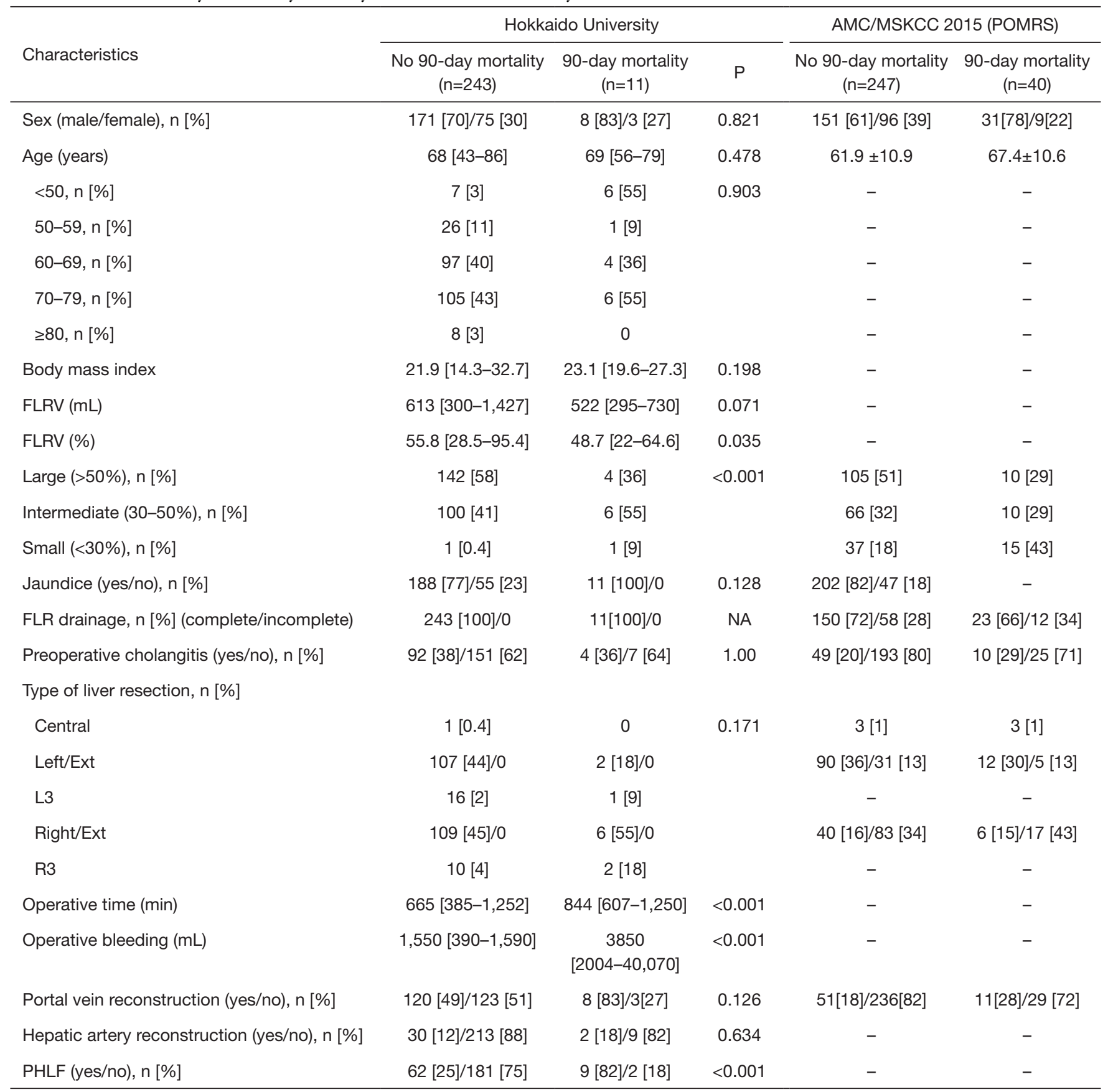

Values are presented as $\mathrm{n}$ or median [range]. NA, not assessed; FLRV, future liver remnant volume; FLR, future liver remnant; central, central hepatectomy; left, left hepatectomy; L3, left trisectionectomy; right, right hepatectomy; R3, right trisectionectomy; PHLF, post-hepatectomy liver failure. 
Table 5 Risks predicted by the mortality risk scores determined in the Hokkaido University series and in the AMC/MSKCC series

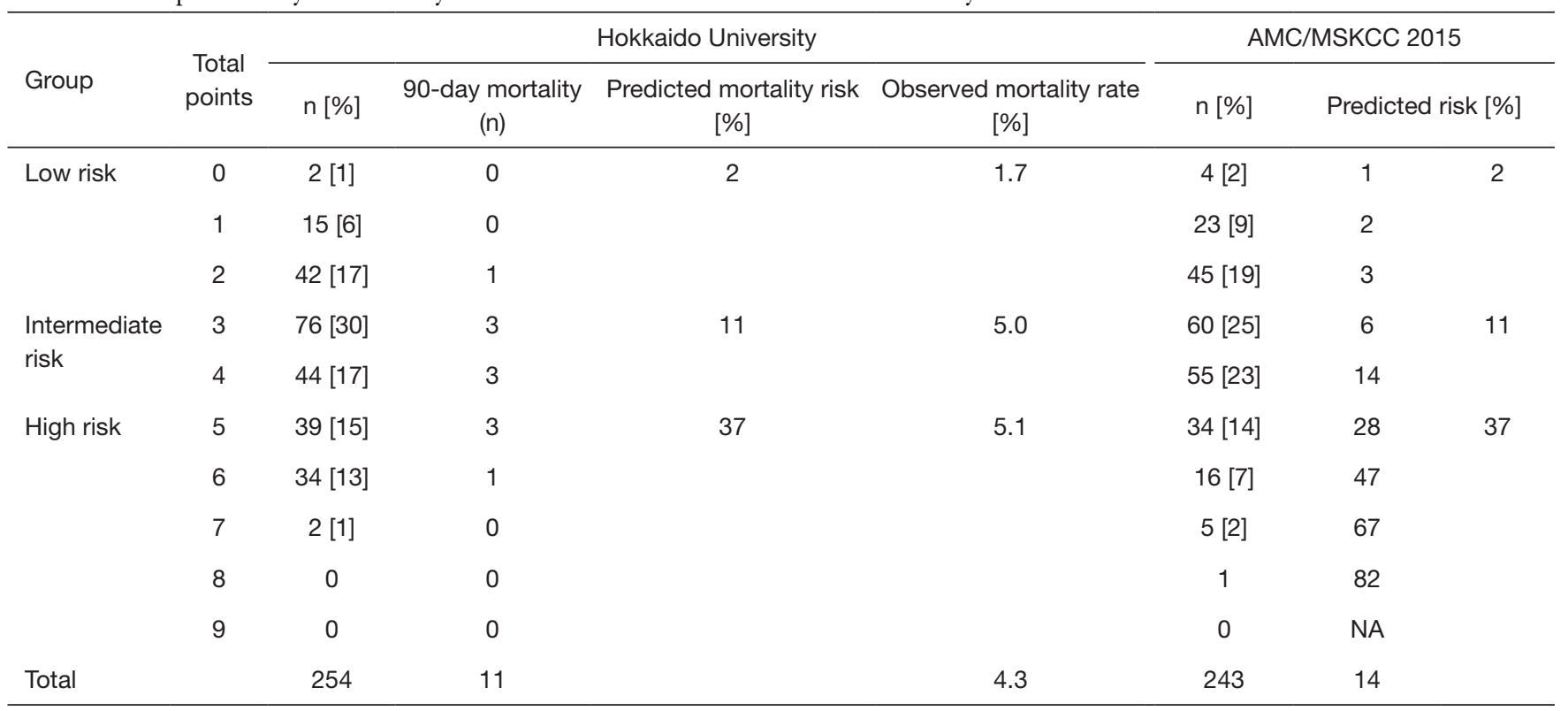

AMC/MSKCC, Academic Medical Center/Memorial Sloan Kettering Cancer Center.

factors did not influence 90-day mortality in the present study. Therefore, this validation study could not reproduce the usefulness of the POMRS in the Hokkaido cohort. This may be explained by differences in the criteria for resection applied in Asia and the West. Very few patients (2/254) had FLRV $<30 \%$ and none had incomplete drainage + FLRV $<50 \%$ in the Hokkaido cohort. However, in the original Western study, 21\% of the patients had FLRV $<30 \%$ and $9 \%$ patients had incomplete drainage + FLRV $<50 \%$, while the mortality rates for these patients were $23 \%$ and $19 \%$, respectively (12).

It is interesting that although both PVR and cholangitis were associated with PHLF in the Hokkaido University cohort (Table 2), these factors were not associated with 90-day mortality (Table 1). Possible reasons why these factors were not associated with 90-day mortality are active application of PVE and complete biliary drainage achieved in the Hokkaido cohort. Several previous reports showed that PVE contributed to an increase of FRLV, and that sustained cholangitis burdened liver regeneration $(26,27)$. While previous reports showed that PHLF was associated with a mortality rate of approximately $50 \%(2,3)$, the present data showed lower mortality rates. As some authors have suggested, although PVE does not decrease the incidence of PHLF as defined by the ISGLS criteria, it could be useful for decreasing PHLF-related mortality $(17,28,29)$. A previous study supports this hypothesis (30).
Other factors of the POMRS (older age and cholangitis) did not affect the 90-day mortality in the Hokkaido University cohort. This potentially leads to a mismatch between predicted mortality risk and observed mortality rate in the present study (Table 5). In general, elderly age is a potential risk factor for postoperative complications after any surgery. High age would be a considerable risk factor, especially in PHCC patients who require major hepatectomy, because aging has been associated with decreased liver function (31). In contrast, a recent study performed at Nagoya University Hospital showed that the surgical results of PHCC for octogenarians did not differ from those of younger generations (32). Our data support the reported results of the Nagoya University group. One possible reason for the age discrepancy could be the strict patient selection at Hokkaido University. In fact, patient selection criteria in the present series were stricter for older patients. Another possible reason might be ethnic differences; one of the longest life expectancies in the world have been recorded in Japan, with an average life expectancy of 82 years.

Because PHLF is the most serious complication after PHCC surgery, left hepatectomy, which leaves the largest FRLV, will be the ideal procedure. Recently, Sugiura et al. (33) showed that preoperative optimization strategies did not affect long term survival in Bismuth type I or II PHCC. On the other hand, previous authors suggested 
that right hepatectomy would be associated with better long-term survival because right hepatic pedicle resection enables better radicality compared with left resections $(14,34,35)$. A recent review however, showed no survival benefit after either right or left resection for PHCC (36).

This study has several limitations. First, the small number of events is associated with statistical uncertainty. In addition, single-centre retrospective cohorts are subject to selection bias. Second, there is an obvious selection bias during the preoperative period. Some patients were denied surgery based on preoperative assessment. According to the selection criteria of Hokkaido University, no patients will undergo PHCC surgery without strict assessments of liver function using ICGK-F and patient's pre-operative status (e.g., serum CRP, nutrition status, and cholangitis). Therefore, there were few patients with FRLV $<30 \%$ and no patients with preoperative bilirubin levels $>50 \mu \mathrm{mol} / \mathrm{L}$ $(2.9 \mathrm{mg} / \mathrm{dL})$. Third, both the original study and this validation study did not evaluate intraoperative factors such as operative bleeding and operative time, which could be influenced by the surgical technique $(3,6,12)$. Fourth, this validation study involved patients with different regional backgrounds. Our previous study showed that several differences existed between Asian and Western patients' backgrounds (13). Moreover, when validating the PHLFS, no patients in the present cohort had a preoperative bilirubin level exceeding $50 \mu \mathrm{mol} / \mathrm{L}$ $(2.9 \mathrm{mg} / \mathrm{dL})$, which was identified as an important factor for predicting PHLF in a previous study (3). It is possible that differences in preoperative management between Asian and Western countries resulted in the low AUC level for the validation of PHLFS. Especially, the lack of patients with preoperative bilirubin levels exceeding $50 \mu \mathrm{mol} / \mathrm{L}$ $(2.9 \mathrm{mg} / \mathrm{dL})$ in the validation set represents one of the most important limitations of this study. Therefore, one of the strongest reasons for the negative results in this study can be attributed to differences between Asia and the West, which makes it difficult to validate PORMS and PHLFS without variations (13).

Furthermore, several clinical questions have not been addressed in this study, such as whether preoperative biliary drainage is necessary in all PHCC patients. A previous report using the POMRS showed that jaundiced patients with no biliary drainage who had FRLV $>50 \%$ and no cholangitis could undergo surgery with no mortality (12). As mentioned, in the present study all patients with jaundice with or without cholangitis underwent biliary drainage. This is not only common practice at Hokkaido University, but also at several Asian high volume centres $(1,37)$. Further, this study could not determine who should undergo PVE for PHCC. The PHLFS suggested that not only patients with small FLRV (FLRV <30\%) but also those with the other three factors (PHLFS 2) should be considered for PVE. In this study, all patients who underwent right hepatectomy or trisectionectomy had undergone PVE. This difference in preoperative preparation is also a major limitation of this study. To solve these issues, we should conduct further validation studies involving international participation and using strict criteria.

Despite these limitations and only partial representation of the PHLFS in the Asian cohort, the present study supports the applicability of the PHLFS in patients with PHCC considered for resection to assess surgical risk and to select patients for PVE. Previous data and the present results support preoperative application of $\mathrm{PVE}$ in patients undergoing trisectionectomy or right hepatectomy with PVR, even if they were categorised as low risk by the PHLFS. Conversely, to determine surgical risk using POMRS in patients with these different regional backgrounds appeared not to be feasible.

In conclusion, this external validation study showed that for PHLFS the threshold for discrimination in an Asian cohort was reached (AUC $>0.6$ ), but it would require optimization of the model before use in clinical practice is acceptable. Application of POMRS was not feasible in the Asian cohort. Further external validation is necessary to confirm whether the PHLFS and POMRS are applicable to Asian cohorts.

\section{Acknowledgments}

Funding: None.

\section{Footnote}

Reporting Checklist: The authors have completed the STROBE reporting checklist. Available at https://hbsn. amegroups.com/article/view/10.21037/hbsn-20-660/rc

Data Sharing Statement: Available at https://hbsn. amegroups.com/article/view/10.21037/hbsn-20-660/dss

Conflicts of Interest: All authors have completed the ICMJE uniform disclosure form (available at https://hbsn. amegroups.com/article/view/10.21037/hbsn-20-660/coif). TMvG serves as an unpaid Deputy Editor-in-Chief of 
Hepatobiliary Surgery and Nutrition. The other authors have no conflicts of interest to declare.

Ethical Statement: The authors are accountable for all aspects of the work in ensuring that questions related to the accuracy or integrity of any part of the work are appropriately investigated and resolved. The present study was performed in accordance with the Declaration of Helsinki (as revised in 2013). The study was approved by the institutional review board of Hokkaido University (No. 018-0429).

Open Access Statement: This is an Open Access article distributed in accordance with the Creative Commons Attribution-NonCommercial-NoDerivs 4.0 International License (CC BY-NC-ND 4.0), which permits the noncommercial replication and distribution of the article with the strict proviso that no changes or edits are made and the original work is properly cited (including links to both the formal publication through the relevant DOI and the license). See: https://creativecommons.org/licenses/by-nc-nd/4.0/.

\section{References}

1. Mizuno T, Ebata T, Nagino M. Advanced hilar cholangiocarcinoma: An aggressive surgical approach for the treatment of advanced hilar cholangiocarcinoma: Perioperative management, extended procedures, and multidisciplinary approaches. Surg Oncol 2020;33:201-6.

2. Franken LC, Schreuder AM, Roos E, et al. Morbidity and mortality after major liver resection in patients with perihilar cholangiocarcinoma: A systematic review and meta-analysis. Surgery 2019;165:918-28.

3. Olthof PB, Wiggers JK, Groot Koerkamp B, et al. Postoperative Liver Failure Risk Score: Identifying Patients with Resectable Perihilar Cholangiocarcinoma Who Can Benefit from Portal Vein Embolization. J Am Coll Surg 2017;225:387-94.

4. Ribero D, Zimmitti G, Aloia TA, et al. Preoperative Cholangitis and Future Liver Remnant Volume Determine the Risk of Liver Failure in Patients Undergoing Resection for Hilar Cholangiocarcinoma. J Am Coll Surg 2016;223:87-97.

5. Yokoyama Y, Ebata T, Igami T, et al. The Predictive Value of Indocyanine Green Clearance in Future Liver Remnant for Posthepatectomy Liver Failure Following Hepatectomy with Extrahepatic Bile Duct Resection. World J Surg 2016;40:1440-7.

6. Kawamura T, Noji T, Okamura K, et al. Postoperative
Liver Failure Criteria for Predicting Mortality after Major Hepatectomy with Extrahepatic Bile Duct Resection. Dig Surg 2019;36:158-65.

7. Kawakami H, Kuwatani M, Onodera M, et al. Endoscopic nasobiliary drainage is the most suitable preoperative biliary drainage method in the management of patients with hilar cholangiocarcinoma. J Gastroenterol 2011;46:242-8.

8. Nagino M. Perihilar cholangiocarcinoma: a surgeon's viewpoint on current topics. J Gastroenterol 2012;47:1165-76.

9. Mizuno T, Ebata T, Yokoyama Y, et al. Major hepatectomy with or without pancreatoduodenectomy for advanced gallbladder cancer. Br J Surg 2019;106:626-35.

10. Yokoyama Y, Nagino M, Nimura Y. Mechanism of impaired hepatic regeneration in cholestatic liver. J Hepatobiliary Pancreat Surg 2007;14:159-66.

11. Olthof PB, Coelen RJS, Wiggers JK, et al. High mortality after ALPPS for perihilar cholangiocarcinoma: case-control analysis including the first series from the international ALPPS registry. HPB (Oxford) 2017;19:381-7.

12. Wiggers JK, Groot Koerkamp B, Cieslak KP, et al. Postoperative Mortality after Liver Resection for Perihilar Cholangiocarcinoma: Development of a Risk Score and Importance of Biliary Drainage of the Future Liver Remnant. J Am Coll Surg 2016;223:321-31.e1.

13. Olthof PB, Miyasaka M, Koerkamp BG, et al. A comparison of treatment and outcomes of perihilar cholangiocarcinoma between Eastern and Western centers. HPB (Oxford) 2019;21:345-51.

14. Kondo S, Hirano S, Ambo Y, et al. Forty consecutive resections of hilar cholangiocarcinoma with no postoperative mortality and no positive ductal margins: results of a prospective study. Ann Surg 2004;240:95-101.

15. Hirano S, Kondo S, Tanaka E, et al. Outcome of surgical treatment of hilar cholangiocarcinoma: a special reference to postoperative morbidity and mortality. J Hepatobiliary Pancreat Sci 2010;17:455-62.

16. Hirano S, Tanaka E, Tsuchikawa T, et al. Oncological benefit of preoperative endoscopic biliary drainage in patients with hilar cholangiocarcinoma. J Hepatobiliary Pancreat Sci 2014;21:533-40.

17. Yokoyama Y, Nishio H, Ebata T, et al. Value of indocyanine green clearance of the future liver remnant in predicting outcome after resection for biliary cancer. Br J Surg 2010;97:1260-8.

18. Noji T, Tsuchikawa T, Okamura K, et al. Concomitant hepatic artery resection for advanced perihilar cholangiocarcinoma: a case-control study with 
propensity score matching. J Hepatobiliary Pancreat Sci 2016;23:442-8.

19. Sakuhara Y, Abo D, Hasegawa Y, et al. Preoperative percutaneous transhepatic portal vein embolization with ethanol injection. AJR Am J Roentgenol 2012;198:914-22.

20. Hirano S, Tanaka E, Shichinohe T, et al. Treatment strategy for hilar cholangiocarcinoma, with special reference to the limits of ductal resection in rightsided hepatectomies. J Hepatobiliary Pancreat Surg 2007;14:429-33.

21. Noji T, Okamura K, Tanaka K, et al. Surgical technique and results of intrapancreatic bile duct resection for hilar malignancy (with video). HPB (Oxford) 2018;20:1145-9.

22. Clavien PA, Strasberg SM. Severity grading of surgical complications. Ann Surg 2009;250:197-8.

23. Rahbari NN, Garden OJ, Padbury R, et al. Posthepatectomy liver failure: a definition and grading by the International Study Group of Liver Surgery (ISGLS). Surgery 2011;149:713-24.

24. Olthof PB, Coelen RJ, van Gulik TM. East or West, Who Grades Liver Failure After Liver Resection for Perihilar Cholangiocarcinoma Best? World J Surg 2017;41:337-8.

25. Takagi T, Yokoyama Y, Kokuryo T, et al. A Clear Difference Between the Outcomes After a Major Hepatectomy With and Without an Extrahepatic Bile Duct Resection. World J Surg 2017;41:508-15.

26. Takagi T, Yokoyama Y, Kokuryo T, et al. Liver regeneration following experimental major hepatectomy with choledochojejunostomy. Br J Surg 2015;102:1410-7.

27. Higuchi R, Yamamoto M. Indications for portal vein embolization in perihilar cholangiocarcinoma. J Hepatobiliary Pancreat Sci 2014;21:542-9.

28. Yokoyama Y, Nagino M, Nimura Y. Mechanisms of hepatic regeneration following portal vein embolization and partial hepatectomy: a review. World J Surg 2007;31:367-74.

Cite this article as: Noji T, Uemura S, Wiggers JK, Tanaka K, Nakanishi Y, Asano T, Nakamura T, Tsuchikawa T, Okamura K, Olthof PB, Jarnagin WR, van Gulik TM, Hirano S. Validation study of postoperative liver failure and mortality risk scores after liver resection for perihilar cholangiocarcinoma. HepatoBiliary Surg Nutr 2022;11(3):375-385. doi: 10.21037/hbsn-20-660
29. Higuchi R, Ota T, Yazawa T, et al. Improved surgical outcomes for hilar cholangiocarcinoma: changes in surgical procedures and related outcomes based on 40 years of experience at a single institution. Surg Today 2016;46:74-83.

30. Nilsson J, Eriksson S, Norgaard Larsen P, et al. Concurrent biliary drainage and portal vein embolization in preparation for extended hepatectomy in patients with biliary cancer. Acta Radiol Open 2015;4:2058460115579121.

31. Cieslak KP, Baur O, Verheij J, et al. Liver function declines with increased age. HPB (Oxford) 2016;18:691-6.

32. Akashi K, Ebata T, Mizuno T, et al. Surgery for perihilar cholangiocarcinoma from a viewpoint of age: Is it beneficial to octogenarians in an aging society? Surgery 2018;164:1023-9.

33. Sugiura T, Okamura Y, Ito T, et al. Left Hepatectomy with Combined Resection and Reconstruction of Right Hepatic Artery for Bismuth Type I and II Perihilar Cholangiocarcinoma. World J Surg 2019;43:894-901.

34. Ratti F, Cipriani F, Piozzi G, et al. Comparative Analysis of Left- Versus Right-sided Resection in Klatskin Tumor Surgery: can Lesion Side be Considered a Prognostic Factor? J Gastrointest Surg 2015;19:1324-33.

35. Ikeyama T, Nagino M, Oda K, et al. Surgical approach to bismuth Type I and II hilar cholangiocarcinomas: audit of 54 consecutive cases. Ann Surg 2007;246:1052-7.

36. Franken LC, Olthof PB, Erdmann JI, et al. Short- and long-term outcomes after hemihepatectomy for perihilar cholangiocarcinoma: does left or right side matter? HepatoBiliary Surg Nutr 2020. doi: 10.21037/hbsn-19-948.

37. Matsuyama R, Mori R, Ota Y, et al. Significance of Vascular Resection and Reconstruction in Surgery for Hilar Cholangiocarcinoma: With Special Reference to Hepatic Arterial Resection and Reconstruction. Ann Surg Oncol 2016;23:475-84. 